\title{
Evolutionary Social Psychology: Prospects and Pitfalls $^{1}$
}

\author{
David M. Buss ${ }^{2}$ \\ University of Michigan
}

The principles of evolutionary psychology and the traditional assumptions of social psychology are highly compatible. Both disciplines trace observed behavioral variability to situational variability. Both assume that psychological mechanisms sensitive to social information are central to causal accounts of social behavior. Questions about the origins and functions of these psychological mechanisms are indispensable for understanding social behavior. Evolutionary psychology provides conceptual tools for addressing these questions. Several pitfalls must be avoided by practitioners of evolutionary social psychology. Specifically, we must jettison notions of genetic determinism and behavioral unmodifiability, eliminate false dichotomies between "genetic" and "learned," and place cross-cultural variability in a sensible theoretical context. Attending to the reliable phenomena discovered by traditional social psychology and the conceptual frameworks provided by modern evolutionary psychology will produce the most informed evolutionary social psychology.

Humans are perhaps the most complexly social of all animals. We live in groups and spend a great deal of time interacting with others, many of

\footnotetext{
${ }^{1}$ This article is an expanded version of a symposium talk presented at the Society for Experimental Social Psychology, October 13, 1990, Buffalo, New York. Preparation of this manuscript was supported by a grant from the National Institute of Mental Health (MH-44206-02). I wish to thank Eugene Burnstein, Martin Daly, Phoebe Ellsworth, James Hilton, Alice M. Isen, and Richard Nisbett for extremely useful suggestions on an earlier version of this paper. I also thank Nancy Cantor, Leda Cosmides, Don Symons, and John Tooby for general discussions that have influenced the ideas expressed about evolutionary social psychology.

${ }^{2}$ Address all correspondence to Professor David M. Buss, Department of Psychology, University of Michigan, 580 Union Drive, Ann Arbor, Michigan 48109-1346.
} 
whom we have relationships with - family members, friends, mates, and co-workers. The field of social psychology has successfully documented a number of fascinating phenomena in social interactions over the past several decades - social loafing (Latane, 1981), obedience to authority (Milgram, 1963), conformity to group judgments (Asch, 1955), the bystander intervention effect (Darley \& Latane, 1968), the in-group/out-group distinction (Tajfel, 1982), the self-serving bias in attribution (Zuckerman, 1979), the effects of physical attractiveness on positive social evaluations (Berscheid \& Walster, 1974), and many others.

These phenomena are interesting and important in their own right, but they typically reside in conceptual isolation both from each other and from a coherent theory of social interaction. Missing from current social psychology is an account of the origins of these phenomena and their functions: Why do humans have psychological mechanisms that cause them to loaf, obey, or conform? What adaptive problems did these phenomena arise to solve? Why should people form relationships that endure over time? Why live in groups at all rather than alone in the woods? These are the questions that evolutionary scientists attempt to address - questions about the origin and functions of our existing psychological mechanisms.

At the same time, many evolutionary thinkers tend to be naive about the cumulative advances that psychology has made. They sometimes underestimate the complexities of social interaction, the detailed psychological machinery needed to process social information, the importance of proximate causes of social behavior, and the cumulative knowledge that has accrued about social processes. These generalizations, of course, do not apply to all evolutionists, nor do the former generalizations apply to all social psychologists. There are some psychologically sophisticated evolutionists, just as there are some evolutionarily sophisticated social psychologists. But few scientists have strong expertise in both disciplines.

In this article, I argue that evolutionary scientists and social psychologists have much to learn from each other. Conjoining the respective strengths of the two disciplines will mutually enrich both fields and create a hybrid field - "evolutionary social psychology." In this article, I argue the following:

1. Social adaptive problems were likely to have imposed the most important selection pressures on humans over the past several million years (see also Brewer \& Caporael, this issue).

2. Evolutionary psychology provides a useful framework for understanding the origins of psychological mechanisms that have been produced by these selection pressures. 
3. Great attention must be directed toward the complex proximate psychological mechanisms or "complex cognitive dynamics" (see Cantor, this issue) that currently underlie social processes, guided by an understanding of the causal processes, principally evolution by natural selection, that created those mechanisms.

4. Fundamental pitfalls and misunderstandings must be avoided by workers in this area, as well as by critics, to arrive at a sensible theoretical account.

\section{BASIC PREMISES OF EVOLUTIONARY SOCIAL PSYCHOLOGY}

Evolutionary perspectives on human behavior are many and varied, often containing premises that contradict one another. Conceptual disagreements are reasonable as theorists struggle with how to think clearly about the issues, go down some blind alleys, correct previous errors, and successively grope toward better theoretical approximations. Because of the diversity of metatheoretical evolutionary assumptions (see, e.g., Brewer \& Caporael, this issue), it is reasonable to spell out which ones are being adopted.

Perhaps the most important conceptual difference among evolutionary scientific views is between sociobiology (what Symons, in press calls "Darwinian anthropology") and the more recent formulations of evolutionary psychology (see Buss, 1991; Symons, in press; Tooby \& Cosmides, 1990). Although sociobiology and evolutionary psychology share the view that humans, including their unique adaptations, have evolved by the process of natural selection as currently understood in inclusive fitness terms (Hamilton, 1964), the two differ in several crucial respects. The key difference hinges on the assumption among many sociobiological treatments (sometimes explicit, sometimes implicit) that humans have evolved domain-general goals or mechanisms to "maximize inclusive fitness" or to act "as if" they were maximizing their inclusive fitness. I've even heard one describe humans as "inclusive fitness maximizing blobs." Evolutionary psychologists, in contrast, believe that it is impossible in principle for natural selection to have created goals or mechanisms of this sort. They see this as a "sociobiological fallacy" - conflating a theory of the causal process that created human psychological mechanisms (inclusive fitness theory) with a theory of the nature of the mechanisms produced by that causal process (mechanisms that strive for inclusive fitness) (Buss, 1991).

Evolutionary psychologists assume instead that natural selection has created a large number of more domain-specific psychological mechanisms, 
each tailored to solving a particular adaptive problem. Sweat glands, for example, exist because those who had them successfully survived and reproduced more than those who did not in the past. But sweat glands simply cool people down. They serve the function of thermal regulation; they do not "strive for fitness" or have fitness as a goal anywhere in the system, either consciously or unconsciously. Evolutionary psychologists view humans not as "fitness strivers" but rather as "adaptation executors" (Tooby \& Cosmides, 1990). To illustrate this difference, consider food preferences. We consume vast quantities of fat, sugar, and salt in fast food restaurants in current environments not because we are striving to replicate our genes, but rather because we are simply activating or "juicing" our evolved taste preferences for these substances. Current execution of these adaptations may even be detrimental to survival and reproduction, and hence "maladaptive" in current environments. These preferences evolved by the process of natural selection, and hence are the end products of a history of differential gene reproduction. But differential gene reproduction is not the "goal" of these preferences; it is the causal process that produced the preferences. The differences between the programs of evolutionary psychologists and that of many sociobiologists extend from basic theoretical differences about what has evolved all the way to empirical methods for testing evolution-based hypotheses, in spite of the shared "general theory" (see below) of evolution by natural selection. Interested readers are referred to Buss (1991), Cosmides and Tooby (1987), and Symons (1990) for extended discussions of the programmatic differences.

There are five basic premises that I will argue provide the foundations for evolutionary social psychology:

1. All Behavior is a Product of Mechanisms Internal to the Person, Combined with Input (External and Internal) that Activates those Mechanisms. Although many of the social phenomena cited above are often viewed as "caused by the situation," a brief thought experiment can be used to show that a sensible causal account requires the invocation of psychological mechanisms internal to organisms (Cosmides \& Tooby, 1987; Symons, 1987). If human beings conform or obey authority, but ants and gorillas do not, in response to identical external inputs, then there must be something fundamental about humans psychological mechanisms that differs from those of ants or gorillas. No behavior can be produced in the absence of these psychological mechanisms. The "situational fallacy" (Buss, 1991) is to assume that, because situational variance can "account for" behavioral variance (e.g., changes in situation can be correlated with changes in the bystander intervention effect), a coherent explanatory account need not involve stable psychological mechanisms residing within the person (e.g., information-processing decision-rules). No behavior can be produced in the absence of mechanisms. 
2. Evolution by Natural Selection is the Only Known Causal Process by Which Complex Species-Typical Functional Mechanisms Can be Created, at Some Fundamental Level of Description. In the history of science, only two alternatives to evolution have been proposed: creationism, the notion that a supreme deity created life in all of its various forms, and seeding theory, the idea that extraterrestrial organisms planted the seeds of life on earth. Creationism and seeding theory, largely incapable of being verified or disproved by observation or experiment, are not scientific theories and lack heuristic value - they lead to no empirical predictions. Although I have seen no formal studies on the matter, my hunch is that very few social psychologists are creationists or seeding theorists. As Symons (1987) phrased it, "we're all Darwinians" in the sense that we believe that humans evolved through a process of natural and sexual selection. The central issue, therefore, is not whether natural and sexual selection created human psychological mechanisms, but rather the nature of the mechanisms that these processes have created (Symons, 1987).

3. Evolved Social Psychological Mechanisms Are Likely to Be Large in Number and Complex in Nature, and Many Will Be Domain-Specific. There are several rationales for this premise, but two will be mentioned, one conceptual and one empirical. Conceptually, the social adaptive problems that humans had to solve in their environment of evolutionary adaptedness were many, complex, and different from one another. A fear of snakes may solve the adaptive problem of avoiding a dangerous environmental hazard, but does nothing to solve the adaptive problem of which foods to consume (e.g., berries and nuts, not twigs or gravel). Similarly, solutions to the problem of "how to attract a mate" do little to solve the problem of "how to socialize children." Different adaptive problems typically select for different adaptive solutions. As Symons (1990) argues, there is no such thing as a general solution because there is no such thing as a general problem.

Mechanisms vary, of course, along many dimensions - some are more domain-general, others are more domain-specific; some are cognitively penetrable, others are cognitively impenetrable; some can be easily overridden by other mechanisms, others are more difficult to override. What constitutes a successful solution to an adaptive problem, however, differs across adaptive domains (e.g., avoiding a snake vs. selecting a mate), individual circumstances (e.g., presence of powerful kin and alliances vs. absence of social resources), different species (humans vs. spiders), different ages (prepubescent vs. adolescent), and different sexes (male vs. female). There can in principle, therefore, be no fully domain-general solution mechanism - one that can be used across all adaptive domains, by all ages, by all sexes, and in all individual circumstances. A carpenter's flexibility comes not from having a single domain-general "all purpose tool" that is 
used to cut, poke, saw, screw, twist, wrench, plane, balance, and hammer, but rather from having many, more specialized, tools. It is the numerousness and specificity of the tools in the entire tool kit that give the carpenter great flexibility, not a highly "plastic" single tool. Similarly, the great flexibility that humans display in dealing with their social environments is due not to having just a few domain-general psychological mechanisms, but rather a large number of complex and specific ones, which can be deployed singly and in combination depending on circumstances. Thus, on conceptual grounds, evolutionary psychologists assume that because (a) adaptive problems are many and distinct, (b) successful solutions to one problem are different from the solutions needed for other problems, and (c) what will be successful depends heavily on species, age, sex, context, and individual circumstances, the solution mechanisms will be numerous and complex. Evolutionary psychology offers a heuristic for identifying some of these human adaptive problems (Buss, 1991).

The second rationale for the complexity-numerousness premise is empirical. In the past 30 years, psychologists have demonstrated again and again violations of proposed principles of domain-generality. In learning theory, for example, violations of equipotentiality have been demonstrated by Garcia and others. Indeed, Garcia's findings at first were so startling to editors and reviewers of the major journals, mainly because they violated domain-general learning principles, that they refused to believe or to publish them until they were replicated numerous times. We now know that some things are extraordinarily difficult to learn, requiring thousands of trials; others can be learned in a single trial. We now know that humans are predisposed to learn some things more readily and rapidly than other things (Seligman \& Hagar, 1972). More people learn fears of snakes, heights, spiders, and darkness, for example, than fears of guns, cars, or electrical outlets, which are currently more hazardous - additional evidence that people are adaptation executors rather than fitness strivers (e.g., more people have fears of snakes than of cars, even though far more people die and are injured by cars than by snakes in modern environments).

The existence of many domain-specific mechanisms, of course, does not rule out the possibility and even likelihood that some mechanisms will be relatively more domain-general such as the capacity for induction, the ability to perceive means-ends relationships, and perhaps the perception of certain forms of covariation. An evolutionary psychological perspective, however, suggests that the human mind cannot be solely composed of such mechanisms - most adaptive problems require more complex and dedicated psychological machinery to successfully solve. Nor will the relatively more domain-general mechanisms be deployed randomly; instead, these mechanisms are co-opted for very specific goals - forming reciprocal alli- 
ances or friendships, selecting mates, achieving or maintaining position within social hierarchies, helping family members, and building coalitions, to name a few central social goals.

Many of empirical findings that are compatible with domain-specificity have been discovered in the social domain, and I'll list just a small sample of them:

a. A nonrandom distribution of fears and phobias such as the "fear of strangers" that emerges reliably between 8 and 24 months of age (Marks, 1987).

b. Child abuse is 40 times greater among preschool children in stepfamilies than "intact" families where there are two genetic parents (this does not imply dedicated mechanisms for child-abuse, but rather may suggest the existence of mechanisms for "discriminative parental solicitude" if other interpretations can be ruled out) (Wilson \& Daly, 1987).

c. Children imitate high-status models much more than low-status models (Bandura, 1977).

d. Some causes of marital dissolution across cultures are highly predictable on evolutionary psychological grounds, centering heavily on infidelity and infertility (Betzig, 1989).

e. Sexual jealousy occurs in all known cultures, and is the leading cause of spousal homicide across cultures (suggesting not dedicated mechanisms for spousal homicide, but rather possibly, male mechanisms involved in "male sexual proprietariness") (Daly \& Wilson, 1988).

f. Men and women show consistent differences in what qualities they desire in potential mates, such as cues to resource investment potential and cues to reproductive value; these differences are closely linked with the social adaptive problems that men and women have confronted in mating contexts; and these differences are highly consistent across cultures (Buss, 1989).

Although not one of these findings tells a definitive story, in the aggregate they are compatible with existence of a large number of specialized social psychological mechanisms. The psychological mechanisms involved in imitating high-status models undoubtedly differ from those involved in avoiding strangers or selecting mates. The empirical evidence, in other words, can be seen as conforming to the conceptual expectations of evolutionary psychologists that numerous mechanisms have evolved because of the large number and extreme diversity of adaptive problems that humans needed to solve in our evolutionary environments. 
4. Many of the Most Important Adaptive Problems for Humans Were Social in Nature. As Brewer and Caporael (this issue) argue, the social group constituted at least one of the crucial "selection environments" for humans. They argue that the cooperative group may have been the primary survival strategy of humans, and this would have selected for adaptations suited for cooperative group living such as cooperativeness, loyalty, and fear of social exclusion (see also Alexander, 1987; Baumeister \& Tice, 1990; Buss, 1990). Individuals whose mechanisms led them to be uncooperative, deviant from group norms, or disloyal presumably would have had more trouble surviving than those with the opposite set of mechanisms, and hence their mechanisms would have been selected against.

But survival is only the beginning. Because natural selection operates by a process of differential reproductive success (not differential survival success), there are many reproductive problems that we had to solve, and many of these are inherently social in nature. Examples include successful intrasexual competition, mate selection, sexual intercourse, mate retention, reciprocal dyadic alliance formation, coalition building and maintenance, parental care and socialization, and extra-parental kin investment (Buss, 1991).

Humans are probably unique in the duration and complexity of social relationships that they form. Humans sometimes form lifelong mating relationships, develop friendships that last for decades, and maintain contact with their brothers, sisters, and other relatives over great expanses of time and distance. Because social adaptive problems were so crucial for human survival and reproduction, many of the most important features of our evolved psychological mechanisms will necessarily be social in nature.

5. Many of the Important Social Adaptive Problems Humans Had to Solve Entailed the Successful Formation of Enduring Social Relationships Such as Mateships, Friendships, Kinships, and Coalitions. Much social psychological research has been described as the "psychology of stranger interaction." Undergraduates who have never met and who will never meet in the future encounter each other for brief periods in social psychological experiments. In everyday life, however, most of our social interactions occur with people with whom we have formed lasting relationships - a fact that is also true among contemporary hunter-gatherer groups, and was undoubtedly true throughout human evolutionary history. Evolutionary psychologists expect, therefore, that some of the most important evolved psychological mechanisms will be tailored to solving the unique adaptive problems associated with such relationships. I will illustrate this with a few examples - reciprocal alliances, mateships, and parent-offspring relationships. First, however, a few words must be said about "levels of analysis" and the testing of evolutionary hypotheses. 


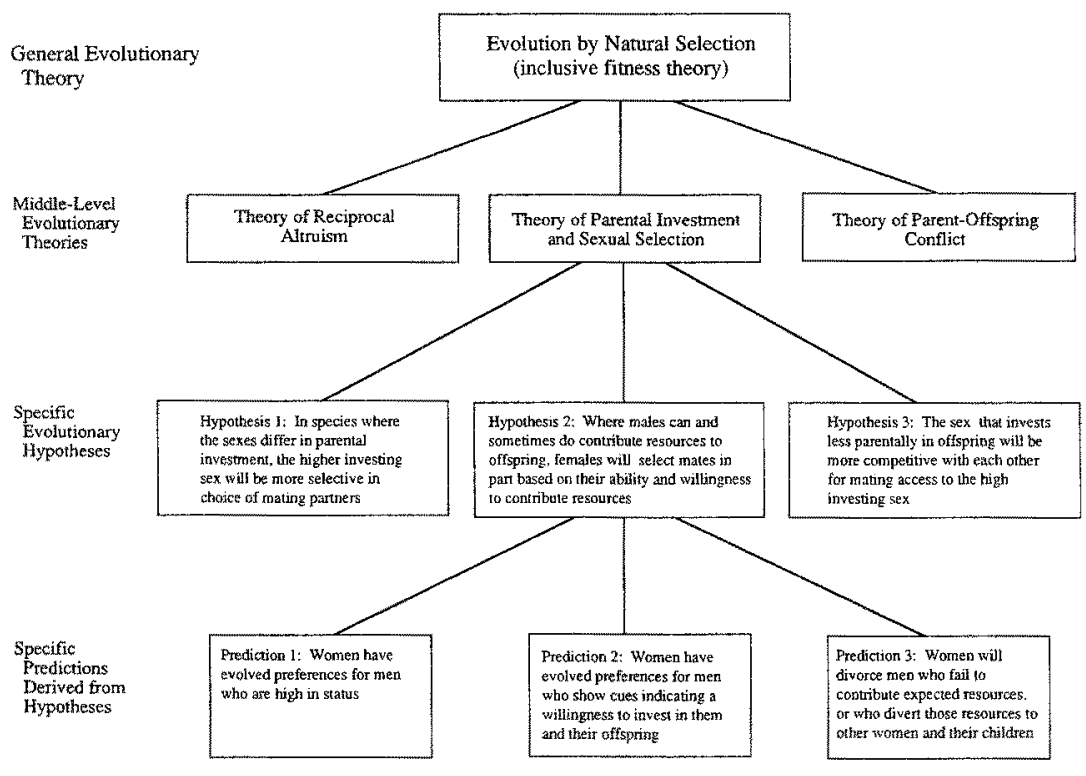

Fig. 1.

\section{LEVELS OF ANALYSIS IN EVOLUTIONARY PSYCHOLOGY}

When I give colloquia about my evolution-based research on human mating strategies, I am sometimes asked questions such as "What evidence would falsify "the theory" " or "Doesn't the existence of people helping total strangers falsify "the theory?" "In order to answer these questions, one must first distinguish among at least four levels of analysis (see Fig. 1). The first level is general evolutionary theory - evolution by natural selection, as understood in its modern form as inclusive fitness theory. Now, at this level, even though general evolutionary theory is called a "theory," it is widely regarded by biologists as so well established that it is simply assumed to be correct in its general outlines, and then work proceeds from that assumption but does not test the assumption, at least not directly. There have been thousands of tests of the general theory. New species can be created in the laboratory using its principles. Evolution by natural selection is the guiding metatheory for the entire field of biology. There are phenomena that could falsify the general theory - if complex life forms were found to be created in time periods too short for natural selection to have operated (e.g., in 7 days), or if adaptations were found on organisms that evolved 
for the benefit of intrasexual competitors or for the benefit of other species. But no one has ever observed or documented such phenomena.

So when an evolutionary psychologist tests an evolutionary proposition, she is not testing "general evolutionary theory," just as when an astronomer tests a particular hypothesis (e.g., about the amount of critical mass in the universe), she is not testing "general relativity theory" with each experiment. That theory is assumed to be true, just as evolution by natural selection is assumed to be true for the present purposes. Because creationism and seeding theory are the only alternatives that have been proposed over the past 130 years, and because there is overwhelming evidence supporting general evolutionary theory, these assumptions are reasonable.

Moving one level down, we find "middle-level evolutionary theories," such as the theory of reciprocal altruism (Trivers, 1971), the theory of parental investment and sexual selection (Trivers, 1972), and many others. These middle-level theories are still fairly broad in that they are theories about entire domains of functioning - for example, the conditions under which parents and their offspring will conflict with one another. These theories are fair game for testing and possible falsification. I'll examine just one to illustrate this point - Trivers' theory of parental investment and sexual selection (see Fig. 1).

This theory, which is itself an elaboration of Darwin's (1871) theory of sexual selection, provides one of the key theoretical ingredients for predicting the operation of mate choice and the operation of intrasexual competition. Leaving aside the logical and reproductive underpinnings of Trivers' theory (which would require a major treatise to spell out), he essentially argued that the sex that invests more in offspring (often, but not always, the female) should be more choosy about mating, whereas the sex that invests less in offspring should be more competitive with members of their own sex for sexual access to the valuable high-investing opposite sex. A woman whose minimum parental investment includes a 9-month gestation period, for example, would be predicted to be more choosy than a man whose minimum parental investment was the contribution of his sperm. Trivers developed additional hypotheses about the precise content of the mate choice-various forms of resources, for example, when certain contextual conditions were met such as resource defensibility, variance in resource holdings among potential mates, and so on. Some of the specific hypotheses derived from Trivers' theory are shown in Fig. 1. And specific predictions can be derived form each of these hypotheses - predictions about evolved psychological mechanisms or behavioral strategies in a particular species.

In testing these predictions, all the conditions of "normal paradigm science" hold. If the predictions do not pan out empirically, then the hy- 
pothesis on which they were based is called into question. If key hypotheses are called into question by several predictive failures, then the truth or value (depending on one's philosophy of science) of the middle-level theory that generated the hypotheses is doubted. Theories that are consistently supported, as for example Trivers' theory of parental investment and sexual selection has been in thousands of empirical studies, are sometimes hailed as major middle-level theories, especially if they prove highly generative of interesting and fruitful avenues of research (and especially if the predictions are specific to them rather than being common to several alternative interpretations). Theories that are not generative or that produce a series of predictive failures are abandoned or replaced by better theories.

This is a highly oversimplified account, of course, and several additional levels of analysis are often involved. Evolutionary psychologists, for example, developed hypotheses about the psychological mechanisms that have evolved in humans to solve particular adaptive problems that humans have faced in ancestral conditions. This often involves a detailed task analysis of the sorts of information-processing mechanisms needed to solve particular adaptive problems, conjoined with an analysis of the relevant ancestral cues that would have been available to organisms in those environments. In my work, for example, I have developed hypotheses about psychological mate preferences of men and women, based in part on Trivers' theory of parental investment. Some have been supported by dozens of studies; a few have not. But for the present purposes, the point is that when one asks "What evidence could falsify the theory?" one must locate the question in the hierarchy of levels. My particular hypothesis about a psychological mechanism could be wrong, even if the theory at one level up that led me to the hypothesis is entirely correct. As in the rest of science, all levels are evaluated by the cumulative weight of the evidence - rarely is a single study definitive one way or the other.

Thus, the empirical methods used by an evolutionary psychologist to evaluate hypotheses and predictions are exactly the same as those used by other psychologists. They include experimental methods (e.g., Cosmides, 1989; Sadalla, Kenrick, \& Vershure, 1987), questionnaire methods (e.g., Buss, 1989; Kenrick, Sadalla, Groth, \& Trost, 1990), analysis of public documents such as homicide statistics or divorce statistics (e.g., Betzig, 1989; Daly \& Wilson, 1988), observational methods (e.g., Buss, 1988; Hill \& Kaplan, 1988), psychophysiological techniques (McGuire \& Troisi, 1987), and many others. Empirical methods, as in other areas of psychology, are tailored to the specific hypothesis being tested. As always, results that transcend several methods are seen as stronger than results limited to a single method. And results found across different populations and cultures are seen as stronger than results limited to a single population or culture. 
Before leaving the "levels of analysis" issue, it should be noted that some evolutionary investigations take as their starting point a phenomenon or an observation, and then try to test hypotheses about its function or about why it evolved. The fact of sexual reproduction itself, for example, remains a major enigma to evolutionary biologists, and there are several competing theories about why sex evolved (e.g., Hamilton, 1980; Tooby, 1982). No previous evolutionary theory "predicted" that sex would exist in advance, but the fact of its existence is fair game for subsequent evolutionary analysis, just as observations in other fields (e.g., observation of galaxies moving away from one another in astronomy) are fair game for subsequent theoretical scrutiny.

To take another example, the existence of the orgasm among women has provoked substantial theoretical interest among evolutionists (e.g., Gould, 1987; Rancour-Laferriere, 1985; Symons, 1979). Several competing evolutionary hypotheses have been advanced, including (1) the paternity confidence hypothesis, (2) the cue to selecting the right male hypothesis, and (3) the "sealing the pair-bond" hypothesis. At least two prominent evolutionists, in contrast, have argued that the female orgasm is not in itself an adaptation, but rather an incidental byproduct, much like male nipples apparently have no function and are incidental byproducts of the fact that females have nipples (Gould, 1987; Symons, 1979). The key point is that it is rare that one can refer to "the" evolutionary hypothesis. Just as there are competing theories in other areas of science, so there are often competing evolutionary hypotheses about the same set of observations. Testing among competing hypotheses, including hypotheses that state that the observed phenomenon is not itself an adaptation, proceeds in the same fashion as "normal paradigm science," and the nature of evidence needed depends on the particular subject of investigation.

\section{SOME EXAMPLES OF EVOLUTIONARY SOCIAL PSYCHOLOGY}

Let me now turn to three specific examples in which evolutionary psychological thinking has been applied to the analysis of social relationships.

1. Reciprocal Alliance Formation or Friendship. Theoretical analyses have shown that tremendous benefits can accrue to individuals who form cooperative reciprocal relationships (Trivers, 1971). Costs are incurred that provide a larger benefit to someone else; at some later time, the recipient of the initial benefit bestows a benefit on the initial giver. Both individuals can gain by this process, above and beyond what they could have gained 
by acting alone. Indeed, humans seem to have reached something of a pinnacle of reciprocal alliance formation, something that has not escaped the attention of social psychologists judging by the importance of social exchange and equity theories (Clark \& Reis, 1988).

One major condition limiting the evolution of reciprocal relationships, however, is the possibility of cheating - when someone fails to reciprocate once he or she has received the benefit from the other person (Cosmides \& Tooby, 1989). Indiscriminate cooperation under conditions that allow cheating would be selected against; such a strategy is vulnerable to exploitation by noncooperators. It would suffer ultimately in the currency of reproductive success, and hence would not evolve. Therefore, a prerequisite for the evolution of enduring reciprocal relationships is solving the adaptive problem of preventing cheating. Cosmides (1989) presents compelling experimental evidence that people possess something like a specialized "look for cheaters" cognitive algorithm that governs the manner in which they reason about social exchange. This is just one of the many social adaptation problems that must be solved in order to form complex reciprocal alliances - others include evolved capacities to recognize different individuals, to remember the histories of one's interactions with different individuals, to communicate one's values to others, and to model the values of other individuals (Cosmides \& Tooby, 1989). Forming reciprocal alliances requires a number of complex psychological mechanisms that are uniquely designed for specialized adaptive problems.

2. Parent-Child Relationships. Parents and children share $50 \%$ of their genes, and so one expects on theoretical grounds that acts of altruism will flow from one to the other without the necessary expectation of reciprocity. What constitutes a "violation" in one type of relationship (e.g., failure to reciprocate) does not constitute a violation in parent-child relationships. Evolutionary psychologists expect that specialized psychological mechanisms will have evolved that are attendant upon the unique adaptive problems that parents have confronted when interacting with their children. One class of solutions to these problems is subsumed by what Daly and Wilson (1988) call "discriminative parental solicitude."

The tremendous surge of love that parents feel toward their children seems to be mitigated by a highly predictable set of conditions that is difficult to account for on any grounds but evolutionary ones: (1) when the parent is not the biological parent, (2) when paternity is uncertain, (3) when the child is deformed or otherwise of poor phenotypic quality, and (4) when circumstances such as poverty, lack of food, or maternal overburdening from too many children render prospects for surviving and flourishing poor (Daly, 1989). It is precisely under these circumstances that infanticide instigated by parents is carried out, at least in traditional societies (Daly \& 
Wilson, 1988; Dickeman, 1975; Minturn \& Stashak, 1982). Without these mitigating circumstances, parents typically invest time, energy, effort, and love that is probably unparalleled in any other type of human relationship.

3. Mating Relationships. In sexually reproducing species such as ours, reproduction cannot occur without mating. In most cultures, the vast majority of individuals do form mating relationships of more than a brief duration (over $90 \%$ of all people get married at some point in their lives). This special social relationship, however, carries unique adaptive problems that are not shared by other forms of social relationships. The possibility of sexual infidelity, at least historically, posed distinctive adaptive problems for men and women. Men whose mates were unfaithful risked investing in children who were not their own. Women whose mates were unfaithful, although not risking analogous maternity uncertainty, nonetheless risked the diversion of a man's resources to other women and their children. There is evidence that "sexual jealousy" evolved as a complex psychological solution to these problems (Daly, Wilson, \& Weghorst, 1982), although as Cantor (this issue) points out, this is no guarantee that jealousy is currently sensible or adaptive.

An interesting point is that this mechanism is irrelevant to solving the adaptive problems associated with friendships or kinships. What constitutes a "violation" in a long-term mateship - having sex with someone else - would not constitute a violation of a friendship, just as failure to reciprocate would constitute a violation of a friendship, but not of a parent-child relationship. Different relationships pose different adaptive problems. What constitutes a successful relationship differs across relationship types. Thus, evolutionary psychologists expect that humans have evolved distinct psychological mechanisms to solve the unique adaptive problems associated with these different types of relationships. Some mechanisms, of course, may operate across contexts - successful status attainment, for example, may simultaneously attract mates and help with the provisioning of children. Selection of a good cooperator might be good for friendships as well as for mateships. Mechanism specificity will occur to the degree that there are specific adaptive demands linked with particular types of relationships.

In sum, humans form many intense, long-duration relationships that differ in their adaptive demands. Long-term relationships undoubtedly constituted human solutions to many of the survival and reproductive problems our ancestors faced. Therefore, the psychology of relationships rightly should occupy a central place in the evolutionary social psychology of humans. Because the adaptive problems posed by different types of relationships were at least partially distinct from each other, solutions to one often do little for solutions to the others. Evolutionary psychologists anticipate 
that there will be numerous social psychological mechanisms that have evolved to deal with these numerous unique complexities.

\section{PITFALLS OF AND PROBLEMS WITH EVOLUTIONARY SOCIAL PSYCHOLOGY}

Evolutionary theory is deceptively simple, and hence easy to misunderstand. This applies to advocates of evolutionary perspectives, as well as to critics. In order to move beyond the stage of strident advocacy and equally strident criticism, it may help to identify some of the most important problems, pitfalls, and misunderstandings linked with this enterprise.

1. Evolutionary Theory Does Not Imply Genetic Determinism or Unmodifiability. Nothing except for death is inevitable and ummodifiable. Not even taxes are inevitable, apparently, if one is rich enough. All adaptations, including evolved psychological mechanisms, develop only in the context of certain environmental inputs. Change those inputs, and you will change the result of development. The last 100 years is replete with examples of environmental interventions that override evolved mechanisms or their manifest expression. We can read a report of a study that links fat consumption with heart attacks, and decide to eat less fat - using our concern with health to attenuate the activation of a food preference that evolved under conditions of caloric scarcity. Thus, it is a misunderstanding of evolutionary psychology to conflate "evolved" with inevitable, intractable, or unmodifiable.

2. Evolutionary Psychology Involves Mechanisms Responsive to Social Context, Environment, and Changes in External Conditions, Not "Instincts" That Rigidly Determine Behavior Regardless of Context. Natural selection and sexual selection produce mechanisms that afford organisms adaptive advantage in dealing with the complex exigencies of their environments. Sweat glands get activated primarily when the external temperature increases; we do not sweat regardless of temperature. In the social domain, the features of the interactional world that humans must track and be responsive to are exponentially more complex than those of the physical world, and change greatly over time, situations, and relationships. We must be able to monitor, for example, our own trajectories and the trajectories of others - mate value trajectories, hierarchical trajectories (who is ascending and descending), shifts in coalitional alliances, and one's own trajectories in all these social relationships. Rigid, inflexible mechanisms imperious to these complexities would be selected against. Evolutionary psychology is centrally about context and environment because these external conditions define the 
adaptive problems humans must solve, and hence the selection pressures for context-sensitive psychological mechanisms.

3. The Dichotomy in the Social Sciences That Contrasts "Genetic" with "Learned" Is a False One, and Must be Jettisoned Before an Understanding of Causal Processes Can Proceed. Evolutionary psychology is often confused with behavioral genetics. In behavioral genetics, researchers often attempt to partition observed variances into "heritable" and "environmental" sources. In that delimited context, it makes sense to speak of something as being "more heritable" or "more environmental," depending on the weight of the evidence.

But from the standpoint of the individual organism, considered as an integrated and unified collection of evolved mechanisms, the phenotype is equally genetic and learned. It makes no sense to separate the two, as they are inseparable in principle. All organisms require DNA, and they equally require environmental input during ontogeny to produce a phenotype. Whether or not something is heritable in the behavioral genetic sense of variance partitioning tends to be irrelevant to evolutionary analyses.

Evolutionary psychology, therefore, has nothing to do with proving that something is genetic rather than learned. Instead, evolutionary psychologists tend to ask about the nature and content of learning, and the form of the learning mechanisms that people have evolved. Why do people learn fear of snakes rather than fear of cars? Why do people learn more from high-status models than from low-status models? Why are people more inclined to learn from the advice of kin than from the advice of a stranger or a competitor? Why do parents socialize their boys differently from their girls (Low, 1989)? How people learn and what they learn are fair game, indeed central game, for evolutionary psychological analysis.

4. Evolutionary Psychology Does Not Imply That What Exists Ought to Exist (Naturalistic Fallacy). The world is filled with things that exist, but that we decide ought not to exist. Many diseases and parasites are "natural" and have evolved, but because of our values, we decide to eliminate or reduce them. What ought to exist is largely a matter that resides with the values of individuals, groups, and societies, and should be clearly separated from the issue of what currently exists. Evolutionary theory at its core is a theory of change, not a theory of stasis. Thus, it would be a grave misunderstanding to use the fact that something evolved as a justification that it must exist or should exist (although values themselves, or their pan-human psychological underpinnings, may warrant analysis as products of evolution by natural selection).

5. Variability Across Culture (Lack of Universality) in Manifest Behavior Does Not Falsify Evolutionary Psychological Hypotheses; Cross-Cultural Variability Often Requires Explanations That Invoke Psychological Mechanisms 
That Track Differing Contextual Input. This is one of the most commonly confused issues. Evolutionary psychologists typically make the crucial distinction between (1) manifest behavior and (2) underlying evolved psychological mechanisms. Much of the observed variability in manifest behavior can be traced to different environmental circumstances impinging on species-typical underlying mechanisms. To use a physical example, calluses are produced by repeated friction to the skin activating the underlying callusproducing mechanisms. If we observe variability across cultures in the number and depth of calluses, we would not conclude that people in some cultures do not have callus-producing mechanisms. Instead, we would trace this manifest variability to environmental variability operating on mechanisms that all humans possess.

Similarly, in the social domain, if we observe variability in mate preferences or in levels of sexual jealousy, an evolutionary psychologist would ask, "Are reliable features of the social or ecological environment being traced by evolved mechanisms that in the aggregate produce average differences between culture?" Preferences for relative plumpness vs. thinness in a mate, for example, vary somewhat across cultures (Symons, 1979). This variability turns out to be highly predictable from the scarcity of food resources in the environment. In cultures where food is scarce, relative plumpness tends to be valued, and indeed is associated with higher status, better health, and a resource-rich kin network in both sexes. In cultures that have greater food abundance, mate preferences tend to run toward the slimmer end of dimension (Symons, 1979), although even in our culture, where fatness is not valued, men generally express a preference for women of medium body builds, not thin builds. Nisbett (this issue) provides an intriguing example of variability in male competitiveness among culture, hypothesizing that different elements in the human strategic repertoire are evoked, depending on the ease of access to food resources.

Thus, it is an error to see cross-cultural variability at the level of manifest behavior as somehow in opposition to evolutionary psychological hypotheses. Cultures vary in at least some ways that are predictable from the reigning features of ecological and social contexts. Cultures provide input into psychological mechanisms, often variable input. This cross-cultural variability gives us an important avenue for exploring the design features of our evolved social psychological mechanisms, many of which evolved precisely to track that variability in contextual input.

The crucial point is that evolutionary psychologists, like many cognitive psychologists, distinguish between evolved mechanisms, environmental input into the mechanisms, and behavior that is a joint product of the input and the operation of the mechanisms on the input. In evaluating cross-cultural evidence, one must be careful about the level of description. Evolu- 
tionary psychologists, for the most part, anticipate universality of evolved psychological mechanisms, so evidence that members of some cultures entirely lacked a particular mechanism (e.g., a sexual jealousy mechanism in males) would falsify a particular evolutionary hypothesis. In contrast, evolutionary psychologists predict that manifest behavior will vary considerably across cultures because of variable input to the mechanisms (e.g., prevalence of jealousy-inducing cues; degree to which males invest in one mate and her offspring). Thus, variability in manifest behavior would not, in itself, falsify an evolutionary hypothesis, unless the behavior varied in ways that contradicted hypotheses about the manner in which the mechanism is proposed to operate.

6. Evolutionary Psychological Hypotheses, If Formulated Precisely, Can Be Tested Empirically. Evolutionary approaches to human behavior are sometime accused of proposing a large number of hypotheses, some of them highly speculative with few empirical tests to back them up. There is undoubtedly some truth to this criticism. Fortunately, it is also becoming less true rapidly - the number of empirical articles on human evolutionary hypotheses has increased dramatically over the past 5 years (e.g., Betzig, 1989; Buss, 1989; Cosmides, 1989; Cunningham, 1986; Daly \& Wilson, 1988; Gangestad \& Simpson, 1990; Kenrick et al., 1990; Langois \& Roggman, 1990; Low, 1989; Pinker \& Bloom, 1990; Snyder, Simpson, \& Gangestad, 1986).

Like hypotheses in all areas of psychology, some are formulated precisely, others sloppily; some are easily testable, others less so; some accrue powerful empirical support, others little or no support. It is important, therefore, to evaluate each evolutionary psychological hypothesis on its own merits. The hypothesis that men will have mechanisms to detect when women are ovulating, for example, is eminently testable and indeed has been tested. But it appears to be wrong - there is no evidence that men can detect ovulation (Symons, 1990). On the other hand, the hypothesis that women value cues to resources and resource acquisition in potential mates has received repeated empirical confirmation from many investigators across many cultures (e.g., Betzig, 1989; Buss, 1989; Townsend, 1989). As noted in the discussion of "levels of analysis," one must be clear about the consequences of a particular predictive confirmation or failure.

Because behavior leaves no fossils, how can we test hypotheses in evolutionary social psychology? Fortunately, living humans probably constitute our most important and revealing source of evidence - all people are living fossils with complex design features that are records of prior selection pressures. Our current psychological mechanisms provide a window into our past (Tooby \& Cosmides, 1990). Just as our callus-producing mechanisms tell us that our ancestors faced the adaptive problem of re- 
peated friction to the skin, the widespread fears of snakes, heights, and strangers tells us that these were likely to have been important hazards for our evolutionary ancestors. Our heightened sensitivity to nonreciprocators suggests the importance of cooperation in human evolutionary history. The great upset evoked by marital infidelity reveals the importance of enduring committed mating relationships. Existing psychological mechanisms - their complex design features and their economy and efficiency for solving certain adaptive problems - provide us with one window through which our selective past can be seen.

Sophistication about testing evolutionary hypotheses, although still far from ideal, is increasing rapidly. It is far from ideal in the sense that some investigators are excessively vague about what mechanisms they are proposing have evolved, often conflating evolved psychological mechanisms and manifest behavior, and resorting instead to imprecise notions of human behavior as being "adaptive." As a consequence, the "evidence" that is sometimes used, such as correlating given characteristics with current reproductive success, is inherently ambiguous and uninformative (Symons, 1990).

But sophistication is improving rapidly, as scientists clarify their thinking and deploy with increasing precision empirical methods for testing specific evolutionary psychological hypotheses. Fortunately for the proposed integration with social psychology, precisely the same methodological tools that social psychologists have developed can be used. What we will change is not our methods, but the questions that are posed - questions about social psychological mechanisms, informed by an understanding of the adaptive problems they may have been designed to solve in the environments in which we evolved.

7. Although Evolution Is a Simple process, Its Application to Human Psychology Will Be Difficult. Evolutionary social psychology will not be easy. We will require a deep understanding of evolutionary biology, including knowledge of the rich set of theories about function. We also need knowledge of human ancestral conditions, knowledge of different cultures including contemporary hunter-gatherer cultures, and knowledge of other species for comparative analysis. Keen insight into the unique social problems that our human ancestors and their ancestors faced will come in part from this broader contextual and historical perspective. Social psychologists can offer sophistication about the information-processing constraints involved in carrying out certain social strategies and methodological sophistication to translate specific evolutionary hypotheses into empirical programs of research. These are formidable hurdles for any scientist, and all the more so for those who lack training in the requisite fields. But there are no real alternatives for developing a deep and lasting evolutionary social psychology. 


\section{CONCLUSIONS}

The assumptions of traditional social psychologists and evolutionary psychologists are highly compatible - they both seek to trace observed variability in manifest behavior to environmental or situational variability. Implicit in most social psychological accounts are underlying species-typical psychological mechanisms that are sensitive to situational information. Evolutionary psychologists add to this scientific quest by trying to be explicit about these psychological mechanisms, the causal processes that produced them, and the functions they were designed to serve.

Social psychology will be enriched, not diminished, by adding this additional layer of causal understanding. As Nisbett (this issue) points out, we have a great deal to gain by making use of the conceptual tools of evolutionary psychology. Questions about the origins of social psychological mechanisms need to be asked, even if our current answers are incomplete: Where did we come from, why do we have the social psychological mechanisms we have, what are their functions, and what are the crucial environmental inputs that activate or deactivate them? Evolutionary psychology will be enriched by attending to the fascinating panoply of reliable phenomena that social psychology has discovered, and to the rich set of methods the field has developed to study them. It is that mutual enrichment that ultimately will produce the most informed evolutionary social psychology.

\section{REFERENCES}

Alexander, R. D. (1987). The biology of moral systems. New York: Aldine de Gruyter.

Asch, S. (1955). Opinions and social pressures. Scientific American, 193, 31-35.

Bandura, A. (1977). Social learning theory. Englewood Cliffs, NJ: Prentice-Hall.

Baumeister, R. F., \& Tice, D. M. (1990). Anxiety and social exclusion. Journal of Social and Clinical Psychology, 9, 165-195.

Berscheid, E., \& Walster, E. (1974). Physical attractiveness. In L. Berkowitz (Ed.), Advances in experimental social psychology (Vol. 7, pp. 157-215). New York: Academic Press.

Betzig, L. (1989). Causes of conjugal dissolution: A cross-cultural study. Current Anthropology, 30, 654-676.

Brewer, M. B., \& Caporacl, L. R. (this issue). Selfish genes vs. sellish people: Sociobiology as origin myth. Motivation and Emotion, 14, 237-243.

Buss, D. M. (1988). The evolution of human intrasexual competition: Tactics of mate attraction. Journal of Personality and Social Psychology, 54, 661-628,

Buss, D. M. (1989). Sex differences in human mate preferences: Evolutionary hypotheses tested in 37 cultures. Behavioral and Brain Sciences, 12, 1-49.

Buss, D. M. (1990). The evolution of anxiety and social exclusion. Journal of Social and Clinical Psychology, 9, 196-201.

Buss, D. M. (1991). Evolutionary personality psychology. Annual Review of Psychology. Palo Alto, CA: Annual Reviews.

Cantor, N. (this issue). Social psychology and sociobiology: What can we leave to evolution? Motivation and Emotion, 14, 245-254. 
Clark, M. S., \& Reis, H. T. (1988). Interpersonal processes in close relationships. Annual Review of Psychology, 39, 609-672.

Cosmides, L. (1989). The logic of social exchange: Has natural selection shaped how humans reason? Cognition, 31, 187-276.

Cosmides, L., \& Tooby, J. (1987). From evolution to behavior: Evolutionary psychology as the missing link. In J. Dupre (Ed.), The latest on the best: Essays on evolution and optimality. Cambridge, MA: MIT Press.

Cosmides, L., \& Tooby, J. (1989). Evolutionary psychology and the generation of culture, Part II. Case Study: A computational theory of social exchange. Ethology and Sociobiology, $10,51-98$.

Cunningham, M. R. (1986). Measuring the physical in physical attractiveness: Quasiexperiments on the sociobiology of female beauty. Journat of Personality and Social Psychology, 50, 925-935.

Daly, M. (1989). Parent-offspring confliet and violence in evolutionary perspective. In R. W. Bell \& N. J. Bell (Eds.), Sociobiology and the social sciences (pp. 25-43). Lubbock: Texas Tech University Press.

Daly, M., \& Wilson, N. (1988). Homicide. New York: Aldine de Gruyter.

Daly, M., Wilson, M., \& Weghorst, S. J. (1982). Male sexual jealousy. Ethology and Sociobiology, 3, 11-27.

Darley, J. M., \& Latane, B. (1968). Bystander intervention in emergencies: Diffusion of responsibility. Joumal of Personality and Social Psychology, 8, 377-383.

Darwin, C. (1871). The descent of man and selection in relation to sex. London: Murray.

Dickeman, M. (1975). Demographic consequences of infanticide in man. Annual Review of Ecology and Systematics, 7, 107-137.

Gangestad, S. W., \& Simpson, J. A. (1990). Toward an evolutionary history of female sociosexual variation. Journal of Personality 58, 69-96.

Gould, S. J. (1987). Freudian slip? Natural History, 2, 14-21.

Hamilton, W. D. (1964). The evolution of social behavior. Joumal of Theoretical Biology, 7, $1-52$.

Hamilton, W. D. (1980). Sex versus non-sex versus parasite. Oikos, 35, 282-290.

Hill, K., \& Kaplan, H. (1988). Tradeoffs in male and female reproductive strategies among the Ache. In L. Betzig, M. Borgerhoff Mulder, \& p. Turke (Eds.), Human reproductive behavior ( $p$ p. 277-306). Cambridge, UK: Cambridge University Press.

Kenrick, D. T., Sadalla, E. K., Groth, G., \& Trost, M. R. (1990). Evolution, traits, and the stages of human courtship: Qualifying the parental investment model. Journal of Personality, 58, 97-116.

Langois, J., \& Roggman, L. A. (1990). Attractive faces are only average. Psychological Science, $1,115-121$.

Latane, B. (1981). The psychology of social impact. American Psychologist, 36, 343-356.

Low, B. S. (1989). Cross-cultural patterns in the training of children: An evolutionary perspective. Journal of Comparative Psychology, 103, 311-319.

Marks, I. M. (1987). Fears, phobias, and rituals. New York: Oxford University Press.

McGuire, M., \& Troisi, A. (1987). Physiological regulation-deregulation and psychiatric disorders. Ethology and Sociobiology, 8, 9-27.

Milgram, S. (1963). Behavioral study of obedience. Joumal of Abnormal and Social Psychology, $67,371-378$.

Minturn, L., \& Stashak, J. (1982). Infanticide as a terminal abortion procedure. Behavior Science Research, 17, 70-90.

Nisbett, R. E. (this issue). Evolutionary psychology, biology, and cultural evolution. Motivation and Emotion, 14, 255-263.

Pinker, S., \& Bloom, P. (1990). Natural language and natural selection. Behavioral and Brain Sciences.

Rancour-Laferriere, D. (1985). Signs of the flesh: An essay on the evolution of hominid sexuality. New York: Mouton de Gruyter.

Sadalla, E. K., Kenrick, D. T., \& Vershure, B. (1987). Dominance and heterosexual attraction. Journal of Personality and Social Psychology, 52, 730-738. 
Seligman, M. E. P., \& Hagar, J. L. (Eds.) (1972). Biological boundaries of leaming. New York: Appleton-Century-Crofts.

Snyder, M., Simpson, J. A., \& Gangestad, S. (1986). Personality and sexual relations. Journal of Personality and Social Psychology, 51, 417-447.

Symons, D. (1979). The evolution of human sexuality. New York: Oxford University Press.

Symons, D. (1987). If we're all Danwinians, what's the fuss about? In C. Crawford, M. Smith, \& D. Krebs (Eds.), Sociobiology and psychology: Ideas, issues, and applications ( $p p$. 121-146). Hillsdale, NJ: Erlbaum.

Symons, D. (1990). On the use and misuse of Darwinism in the study of human behavior. In J. Barkow, L. Cosmides, \& J. Tooby (Eds.), The adapted mind: Evolutionary psychology and the generation of culture.

Tajfel, H. (1982). Social psychology of intergroup relations. In M. R. Rosensweig \& L. W. Porter (Eds.), Annual review of psychology (Vol. 33). Palo Alto, CA: Annual Reviews.

Tooby, J. (1982). Pathogens, polymorphism, and the evolution of sex. Journal of Theoretical Biology, 97, 557-576.

Tooby, J., \& Cosmides, L. (1990). The past explains the present: Emotional adaptations and the structure of ancestral environments. Ethology and Sociobiology, 11, 375-424.

Townsend, J. M. (1989). Mate selection criteria: A pilot study. Ethology and Sociobiology, 10, 214-254.

Trivers, R. (1971). The evolution of reciprocal altruism. Quarterly Review of Biology, 46, 35-57.

Trivers, R. (1972). Parental investment and sexual selection. In B. Campbell (Ed.), Sexual selection and the descent of man: 1871-1971 (pp. 136-179). Chicago: Aldine.

Wilson, M., \& Daly, M. (1987). Risk of maltreatment of children living with step-parents. In R. J. Gelles \& J. B. Lancaster (Eds.), Child abuse and neglect. Hawthorne, NY: Aldine de Gruyter.

Zuckerman, M. (1979). Attribution of success and failure revisted, or: The motivational bias is alive and well in attribution theory. Journal of Personality, 47, 245-287. 